\title{
CONFÉRENCE
}

\section{Chirurgie pré-implantaire : les Techniques alternatives aux greffes autogènes. Indications et critères de choix des biomatériaux Dr Christian Mole}

Nancy

En chirurgie pré-implantaire, l'os autogène reste aujourd'hui le " gold-standard » car il offre une fiabilité incomparable dans le pronostic et les suites opératoires des techniques reconstructives. Toutefois, il procure les inconvénients qu'imposent un prélèvement qui est souvent lié à des difficultés techniques pour le praticien, à des douleurs entretenues pour le patient, à une morbidité certaine et parfois même à des complications immédiates ou tardives sur les sites donneurs.

Pour limiter ces effets négatifs, le recours à des biomatériaux de substitution osseuse s'est imposé depuis de nombreuses années. La recherche fondamentale, puis l'industrie nous ont sans cesse abreuvés de nouveaux biomatériaux substitutifs que la recherche clinique s'est efforcée par la suite de codifier dans leurs protocoles d'utilisation, parfois avec succès, parfois au contraire en générant des effets délétères pour le comportement cicatriciel osseux.

Aujourd'hui le recours à des techniques chirurgicales combinées associant optimisation cicatricielle, biomatériaux et régénération osseuse guidée s'impose au quotidien.

Cette communication essentiellement clinique tentera de répondre à deux questions :

- que pouvons aujourd'hui retenir d'un recours raisonné à ces techniques alternatives aux greffes autogènes?

- comment y recourir à bon escient au sein de nos plans de traitement pour optimiser les résultats esthétiques et biomécaniques en implantologie, ou pour accroitre le pronostic et le confort pour nos patients? 